\title{
A new twist in the microRNA pathway: Not Dicer but Argonaute is required for a microRNA production
}

\author{
Gabriel D Bossé ${ }^{1}$, Martin J Simard ${ }^{1}$ \\ ${ }^{1}$ Laval University Cancer Research Centre, Hôtel-Dieu de Québec (CHUQ), Quebec City, Québec G1R 2J6, Canada \\ Cell Research (2010) 20:735-737. doi:10.1038/cr.2010.83; published online 15 June 2010
}

Found in all metazoans, microRNAs or miRNAs are small non-coding RNA of $\sim 22$ nucleotides in length that completely reshaped our understanding of gene regulation. This new class of gene regulator is mostly transcribed by the RNA polymerase II producing a long stem-loop structure, called primary- or pri-miRNA, that will first be processed in the cell nucleus by a multiprotein complex called microprocessor to generate a shorter RNA structure called precursor- or pre-miRNA. The precisely processed pre-miRNA will next be exported into the cytoplasm by Exportin 5 and loaded onto another processing machine containing the ribonuclease III enzyme Dicer, an Argonaute protein and other accessory cellular factors (Figure 1A; [1]). Dicer will mediate the cleavage of the pre-miRNA to form the mature miRNA that will then be bound by the Argonaute protein to form, most likely with other cellular factors, the effector silencing complex or RISC able to modulate protein expression upon binding by sequence complementarity to the specific region of $3^{\prime}$ untranslated region (UTR) of mRNA to alter protein synthesis.

In animals, some Argonaute proteins can cleave RNA molecules while others have lost their catalytic activity and participate in a gene regulatory mechanism

Correspondence: Martin J Simard

E-mail: Martin.Simard@crhdq.ulaval.ca

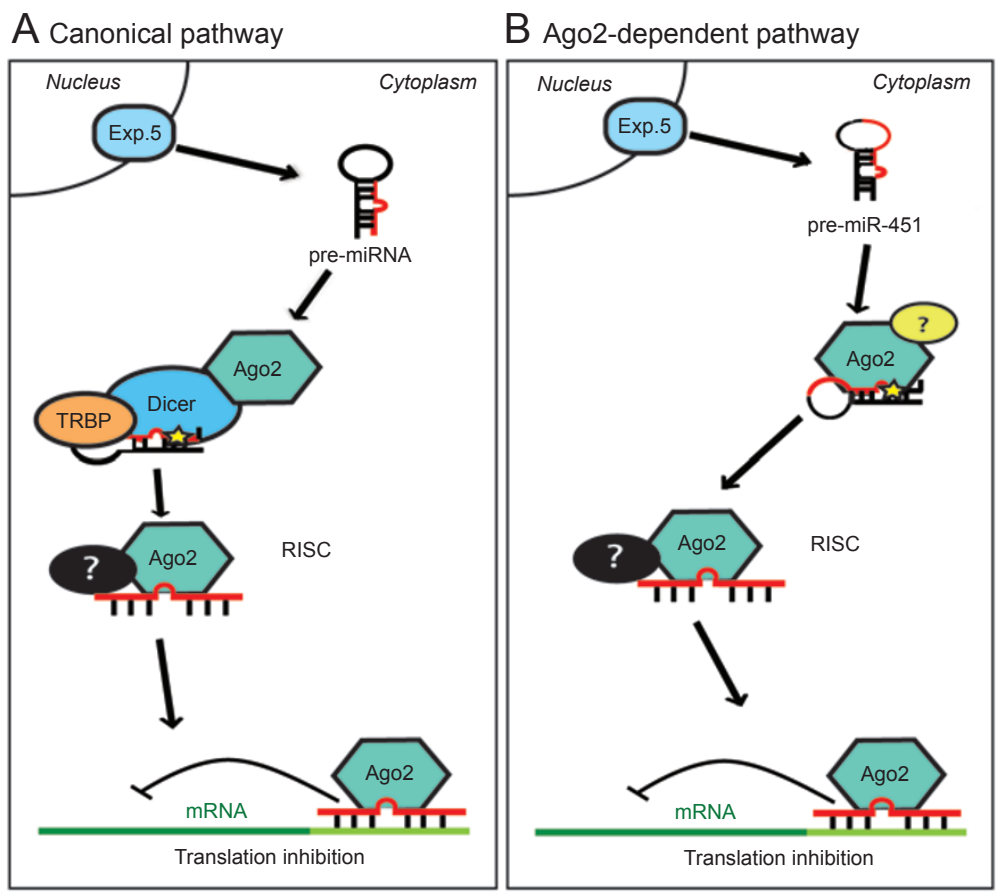

Figure 1 (A) Canonical microRNA biogenesis. In mammals, the pre-miRNA is loaded onto a multiprotein complex consisting minimally of Dicer, Tar RNA Binding Protein (TRBP) and Ago2. The RNase III enzyme Dicer processes the precursor by cleaving the stem-loop to produce the mature miRNA. Ago2 bind this miRNA to form the RISC complex to target specific mRNA. (B) Ago2-dependent microRNA biogenesis. For miR-451, the precursor is loaded directly onto Ago2, and this Argonaute cleaves the precursor RNA of this miRNA. The mature miR451 is bound by Ago2 to form the RISC complex that targets erythropoieticspecific mRNAs.

that does not require RNA cleavage [2]. This family of proteins possesses four structural domains: the $\mathrm{N}$-terminus, PAZ, MID and PIWI domains. The PAZ domain is implicated in the binding of single-stranded RNA while the PIWI domain confers the endonucleolytic activity of Argonaute proteins and requires coordination of divalent cations by Aspartate-Aspartate-Histidine (DDH) motif for catalysis. Interestingly, all four Argonautes found in mammals can form 
a functional RISC but only Ago 2 diplays an endonuclease activity $[3,4]$.

The discovery of a new class of small RNAs in mammal oocytes [5] and embryonic stem cells [6] that have the potential to cleave complementary RNA sequences has provided part of the reason why Ago2 has conserved its enzymatic activity. However, the deletion of Ago2 does not only lead to sterile animals but lethality is observed during gestation $[3,7,8]$ suggesting a role of Ago2 apart of the female germline.

In order to understand the catalytic function of Ago2 in animals, the Hannon's group generated a mouse in which they replaced the endogenous allele by a catalytically inactive Ago2 carrying mutation in the DDH motif (Ago ${ }^{\mathrm{ADH}}$ ) [9]. They observed that the animal underwent a normal embryogenesis but died within a few hours after birth and displayed severe sign of anemia. These embryos have an important reduction in red blood cell caused by a defect in the maturation of erythroid cells. These results represent the first evidence that the catalytic domain of Ago 2 is essential for the survival of mammals.

To next determine the molecular phenotypes of Ago $2^{\mathrm{ADH}}$ mice, Cheloufi and colleagues performed deep sequencing analysis of short RNAs in the liver, one of the organ contributing to erythroid cells maturation in fetus [9]. Among all the miRNAs expressed in these tissues, they surprisingly observed that only one miRNA in normal fetal liver, miR-451, is strongly decreased in mice carrying catalytically inefficient Ago2. Since the expression of the precursor miRNA form of miR-451 was not affected, the authors concluded that the mutation of Ago2 most likely impact the maturation of miR-451. A careful analysis of the miR-451 precursor and mature RNA structure reveals significant differences between miR-451 and others miRNAs. At first, they noted that the pre-miR-451 is shorter by 17 nucleotides than most of pre-miRNAs, making this molecule unlikely a Dicer substrate since this enzyme cannot recognize and process short pre-miRNA efficiently [10]. Second, they also observed that the mature miR-451 includes the sequence of the loop region and expands into the complementary strand of the precursor. The authors concluded from these findings that the biogenesis of miR-451 must be unusual, most likely not inferring Dicer to process the precursor structure and produce mature miRNA.

To further test this hypothesis, the authors used embryonic stem cells carrying a conditional Dicer allele. Using that system, they observed that the production of mature miR-451 was not affected by the absence of Dicer whereas the loss-of-function of Dicer caused a strong decrease of other miRNA levels. To confirm this observation, the authors performed an in vitro assay to measure the efficiency of a recombinant Dicer protein to processed pre-miRNA into mature miRNA. While a pre-let-7 RNA molecule was effectively process into a mature miRNA form, recombinant Dicer was unable to generate the mature miR-451 from its precursor. These results clearly indicate that in contrast to other miRNAs the biogenesis of miRNA miR-451 is Dicer independent and thus, the Argonaute protein Ago2 could be responsible for the biogenesis of this miRNA.

To confirm this hypothesis, the authors performed a thorough analysis of the small RNA species sequenced from the Ago $2^{\mathrm{ADH}}$ animal fetal liver [9]. They observed a significant accumulation of the pre-miR-451 and confirmed the absence of mature miR-451 in Ago ${ }^{\mathrm{ADH}}$ samples. The immunoprecipitation of the Ago2 complex from a fetal liver homogenate of the transgenic animal revealed that pre-miR-451 can bind to the mutated Argonaute. Finally, in vitro assays using both wild-type and catalytically inactive affinity purified Ago2 proteins confirm that Ago2 can successfully process the pre-miR-451 to generate miR-451 and this miRNA is also able to be loaded onto Ago2 to form the RISC complex.

The recent work of Cheloufi and colleagues represents an important breakthrough in the understanding of the catalytically competent Argonaute Ago2 and thus in the miRNA biogenesis in animals. In addition to clearly demonstrating the biological relevance of Argonaute cleavage in the maturation of miRNAs as previously proposed [11-14], this study represents the first evidence of a miRNA maturation that is independent of Dicer. This unexpected miRNA biogenesis seems to be conserved among species as recently reported in Zebrafish by Cifuentes and colleagues [15]. The implication of Ago2 in the processing of pre-miRNA provides interesting evidence of the importance of the endonuclease activity of this Argonaute. It will be next interesting to know how many animal miRNAs require Ago2 to be produced. Since the cleavage of pre-miR-451 by Ago2 should generate a 30 nucleotides long RNA specie, it will be of great interest to understand how this miRNA precursor can be trimmed precisely to generate a 21-23 nucleotides long functional miRNA. To conclude, this exciting work is yet another example that clearly illustrates that we should be extremely cautious of making "general" rule in biology; especially in a fast pacing field like the miRNA-mediated silencing.

\section{References}

1 Winter J, Jung S, Keller S, Gregory RI, Diederichs S. Many roads to maturity: microRNA biogenesis pathways and their regulation. Nat Cell Biol 2009; 11:228-234.

2 Hutvagner G, Simard MJ. Argonaute proteins: key players in RNA silencing. Nat Rev Mol Cell Biol 2008; 9:22-32.

3 Liu J, Carmell MA, Rivas FV, et al. Argonaute 2 is the catalytic engine of mammalian RNAi. Science 2004; 305:1437-1441.

4 Meister G, Landthaler M, Patkaniowska A, et al. Human Argonaute2 mediates RNA cleavage targeted by miRNAs 
and siRNAs. Mol Cell 2004; 15:185197.

5 Tam OH, Aravin AA, Stein P, et al. Pseudogene-derived small interfering RNAs regulate gene expression in mouse oocytes. Nature 2008; 453:534538.

6 Babiarz JE, Ruby JG, Wang Y, Bartel DP, Blelloch R. Mouse ES cells express endogenous shRNAs, siRNAs, and other Microprocessor-independent, Dicer-dependent small RNAs. Genes Dev 2008; 22:2773-2785.

7 Morita S, Horii T, Kimura M, et al. One Argonaute family member, Eif2c2 (Ago2), is essential for development and appears not to be involved in DNA methylation. Genomics 2007; 89:687696.
8 Alisch RS, Jin P, Epstein M, Caspary T, Warren ST. Argonaute2 is essential for mammalian gastrulation and proper mesoderm formation. PLoS Genet 2007; 3:e227.

9 Cheloufi S, Dos Santos CO, Chong MM, Hannon GJ. A dicer-independent miRNA biogenesis pathway that requires Ago catalysis. Nature $2010 \mathrm{Apr}$ 27. doi:10.1038/nature09092

10 Siolas D, Lerner C, Burchard J, et al. Synthetic shRNAs as potent RNAi triggers. Nat Biotechnol 2005; 23:227231.

11 Miyoshi K, Tsukumo H, Nagami T, Siomi H, Siomi MC. Slicer function of Drosophila Argonautes and its involvement in RISC formation. Genes Dev 2005; 19:2837-2848.
12 Leuschner PJ, Ameres SL, Kueng S, Martinez J. Cleavage of the siRNA passenger strand during RISC assembly in human cells. EMBO Rep 2006; 7:314320.

13 Matranga C, Tomari Y, Shin C, Bartel DP, Zamore PD. Passenger-strand cleavage facilitates assembly of siRNA into Ago2-containing RNAi enzyme complexes. Cell 2005; 123:607-620.

14 Rand TA, Petersen S, Du F, Wang X. Argonaute2 cleaves the anti-guide strand of siRNA during RISC activation. Cell 2005; 123:621-629.

15 Cifuentes D, Xue H, Taylor DW, et al. A novel miRNA processing pathway independent of Dicer requires Argonaute 2 catalytic activity. Science 2010 May 6. doi:10.1126/science.1190809 